\title{
Initial Single-Institution Experience With a Novel Robotic- Navigation System for Thoracolumbar Pedicle Screw and Pelvic Screw Placement With 643 Screws
}

\author{
DEEPTEE JAIN, MD, JORDAN MANNING, BA, ELIZABETH LORD, MD, THEMISTOCLES \\ PROTOPSALTIS, MD, YONG KIM, MD, AARON J. BUCKLAND, MBBS, FRACS, JOHN BENDO, MD, \\ CHARLA FISCHER, MD, JEFFREY GOLDSTEIN, MD \\ Division of Spine Surgery, Department of Orthopaedic Surgery, New York University, New York, New York
}

\begin{abstract}
Background: Robotic-guided navigation systems for pedicle screw placement has gained recent interest to ensure accuracy and safety and diminish radiation exposure. There have been no published studies using a new combined robotics and navigation system (Globus ExcelsiusGPS system). The purpose of this study was to demonstrate safety with this system.

Methods: This is a case series of consecutive patients at a single institution from February 1, 2018, to August 31, 2018. All patients who had planned placement of thoracic and lumbar pedicle screws using the combined roboticsnavigation system were included. Chart review was performed for operative details. A subgroup analysis was performed on patients with postoperative computed tomography (CT) scans to assess screw placement accuracy using the Gertzbein and Robbins system. Acceptable pedicle screw position was defined as grade A or B.

Results: One hundred six patients were included, with 636 pedicle screws, 6 iliac screws, and 1 S2AI screw. Five cases were aborted for technical issues. In the remaining 101 patients, 88 patients had screws placed using preoperative CT planning and 13 patients using intraoperative fluoroscopy planning. All screws except for 5 pedicle screws in 2 patients were placed successfully using the robot $(99 \%)$. These 5 pedicle screws were placed by converting to a fluoroguided technique without robotic assistance. Eighty-six patients had screws placed using a percutaneous technique, and 15 patients had screws placed using an open technique. Ninety-eight patients underwent interbody placement: 28 anterior lumbar interbody fusions (ALIFs), 12 lateral lumbar interbody fusions (LLIFs), and 58 transforaminal lumbar interbody fusions (TLIFs). All ALIFs and LLIFs were performed prior to placement of the screws. Four LIF patients had screws placed in the lateral position. No patients had screw-related complications intraoperatively or postoperatively, and no patients returned to the operating room for screw revision. Thirteen patients underwent postoperative $\mathrm{CT}$ for various reasons. Of the 66 pedicle screws that were examined with postoperative CT, all screws $(100 \%)$ had acceptable position.

Conclusion: This study demonstrates that the combined robotics and navigation system is a novel technology that can be utilized to place pedicle screws and pelvic screws safely and has the potential to reduce screw-related complications.
\end{abstract}

Level of Evidence: 4 (case series).

New Technology

Keywords: robotics, pedicle screw placement, spine surgery, navigation, screw accuracy, complications

\section{BACKGROUND}

Posterior lumbar spine fusion is one of the most commonly performed procedures, with over 400,000 performed in the United States in 2008. ${ }^{1}$ Spinal instrumentation is a critical step in thoracolumbar spine surgery for a variety of spinal conditions. Traditionally, free-hand techniques have been employed; however, misplacement of screws is common, estimated at 5\%. Such misplacement risks neurologic or vascular injury, as well as biomechanical compromise. ${ }^{2,3}$ Robotics offers the potential to decrease the risks of spinal instrumentation using computed tomography (CT)-guided navigation and robotic arm placement to precisely guide screw placement.

Prior work examining accuracy of screw placement using robotics is promising, with rates ranging from $85 \%$ to $100 \% .^{4}$ Almost all prior studies have been performed using the Mazor system. The 


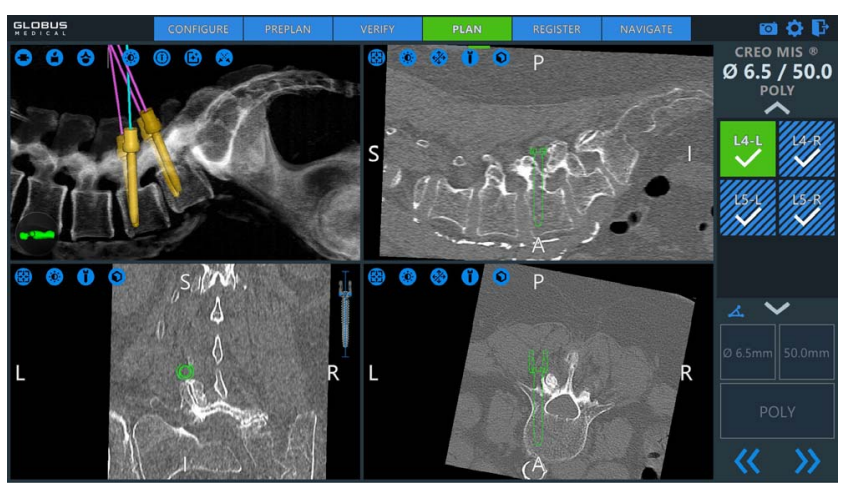

Figure 1. Preoperative planning of screw trajectories. The surgeon positions each screw in the desired trajectory in all 3 planes. The upper left hand quadrant is a ghost reconstruction with the virtual screws superimposed.

purpose of this nonindustry funded study was to describe a single institution's experience with novel robotic navigated system in placing thoracolumbar and pelvic fixation, using screw-related complications as the primary outcome measure.

\section{METHODS}

This is a case series of consecutive patients at a single institution from February 1, 2018, to August 31, 2018. All patients who had planned placement of thoracic and lumbar pedicle screws using combined robotics and navigation system with ExcelsiusGPS (Globus, Audubon, Pennsylvania) were included in the study. As this was a study regarding perioperative complications, minimum follow-up was 1 day.

Chart review was conducted for demographic variables and operative details, with specific attention to system failures and perioperative complications. Variables are described using counts, means, and standard deviations.

A subgroup analysis was performed on patients with postoperative CT scans performed for various reasons. Postoperative CT scans were examined on the sagittal and axial cuts. The cut with the largest deviation of the screw from the pedicle was chosen. Accuracy of screw placement was determined using the Gertzbein and Robbins system (GRS). Screws completely within the pedicle were grade $\mathrm{A}$, a breach $<2 \mathrm{~mm}$ was grade $\mathrm{B}, 2$ to $4 \mathrm{~mm}$ grade $\mathrm{C}$, 4 to $6 \mathrm{~mm}$ grade $\mathrm{D}$, and $>6 \mathrm{~mm}$ grade $\mathrm{E}$. Both grades A and B were defined as acceptable. ${ }^{5}$ All radiographic assessments were performed by a spine surgery fellow.

\section{Surgical Workflow}

For all cases except those in the single position lateral, the patient is positioned in the prone position on a 6-post Jackson frame. The system's fiducial marker, called the dynamic reference base, and the surveillance marker are placed in each posterior superior iliac spine (PSIS) through a stab incisions. The fluoroscopy registration fixture is attached to the image intensifier. Patients underwent either preoperative CT planning workflow or intraoperative fluoroscopy planning workflow.

In the preoperative CT planning workflow, the patient undergoes a preoperative CT scan, which is then loaded into the ExcelsiusGPS system. The screw trajectories are planned out preoperatively in all 3 planes (Figure 1). Intraoperatively, anteriorposterior (AP) and lateral fluoroscopic images of the levels to be instrumented are obtained. A merge is performed between the fluoroscopic images and the preoperative CT scan. The surgeon verifies that ghost reconstruction with the planned screw trajectories from the CT scan matches the fluoroscopically obtained images. The robot is then draped sterilely, wheeled into the field, and locked securely. The robotic arm aligns the end effector to the planned trajectory once the desired screw label is selected. The screw hole is drilled and tapped, and the screw is then placed. The instruments are displayed as they are advanced through the end effector. Figure 2 demonstrates screw placement through the robotic arm, with visualization of the entry of the screw on the screen.

In the fluoroscopic imaging workflow, perfect AP and lateral images of the levels to be instrumented are obtained. After registration is completed, landmark check is performed using a navigated verification probe. The screw trajectories are then planned. Similar to the CT planning workflow, the robotic arm then aligns the end effector to the desired screw trajectory, and the screws are drilled, taped, and placed.

For the single-position lateral patients, the patient is positioned in the lateral position as previously described for the LLIF. After the completion of the LLIF, the table is tilted away from the surgeon approximately 15 degrees. The placement of the dynamic reference base, use of the robot, and placement of the screws then proceeds as described above. Figure 3 shows intraoperative photos of positioning and screw placement in the lateral position. 


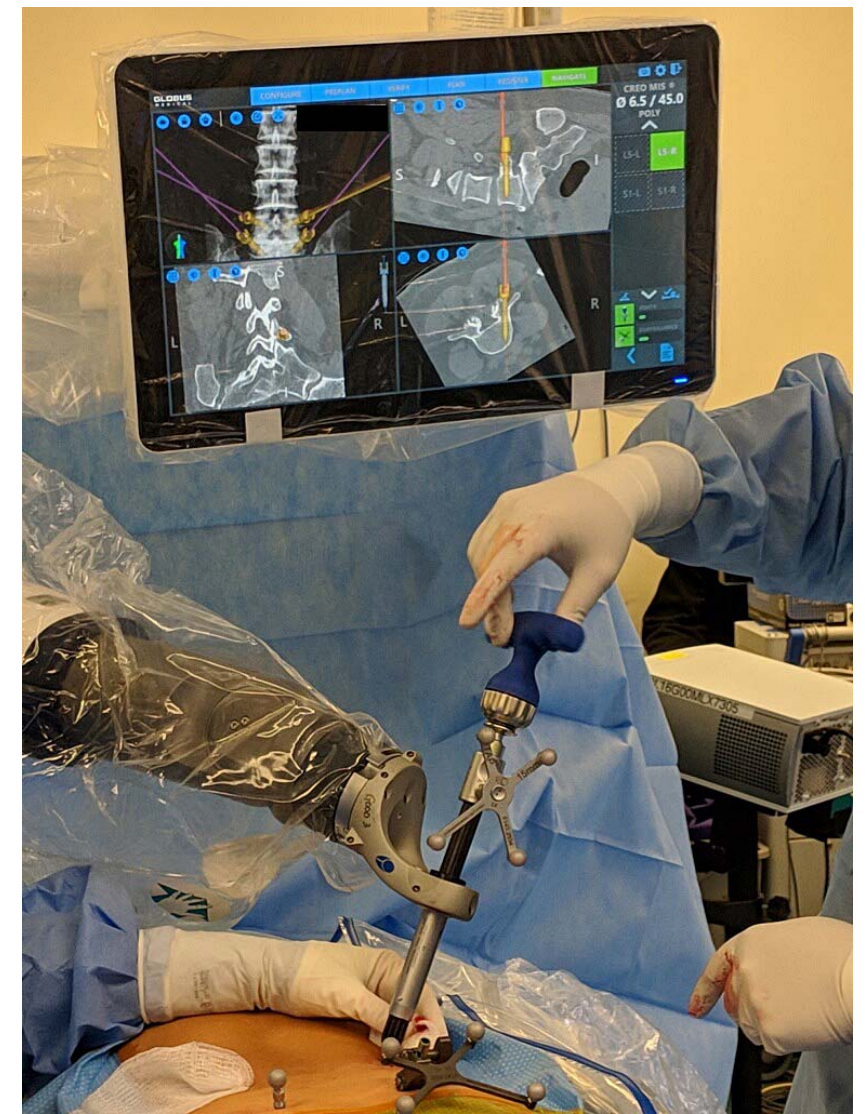

Figure 2. Intraoperative photo of screw insertion through the robotic arm. The screw is displayed overlying the CT on the display.

\section{RESULTS}

There were 106 patients included in the study. Average age was $59.3 \pm 14.8$ years. Fifty-seven were female, 49 were male. Average body mass index (BMI) was $29.2 \pm 6.2 \mathrm{~kg} / \mathrm{m}^{2}$.

Five cases were aborted prior to any screw placement. Two cases were due to technical failure as follows: (1) the end effector could not be validated, (2) the fluoroscopy machine was not appropriately communicating with robot, thought to be due to a hardware failure of the cord. Three cases were due to difficulty merging the preoperative CT scan with intraoperative fluoroscopy as follows: (1) in 2 cases, this was thought be secondary to high patient BMI, (2) in 1 case, this was thought to be due to change in alignment after TLIF.

In the remaining 101 patients, there were 636 pedicle screws, 6 iliac screws, and 1 S2AI screw placed. Eighty-eight patients had screws planned using preoperative CT workflow; 13 patients had screws planned using intraoperative fluoroscopy workflow. All screws except for 5 pedicle screws (2

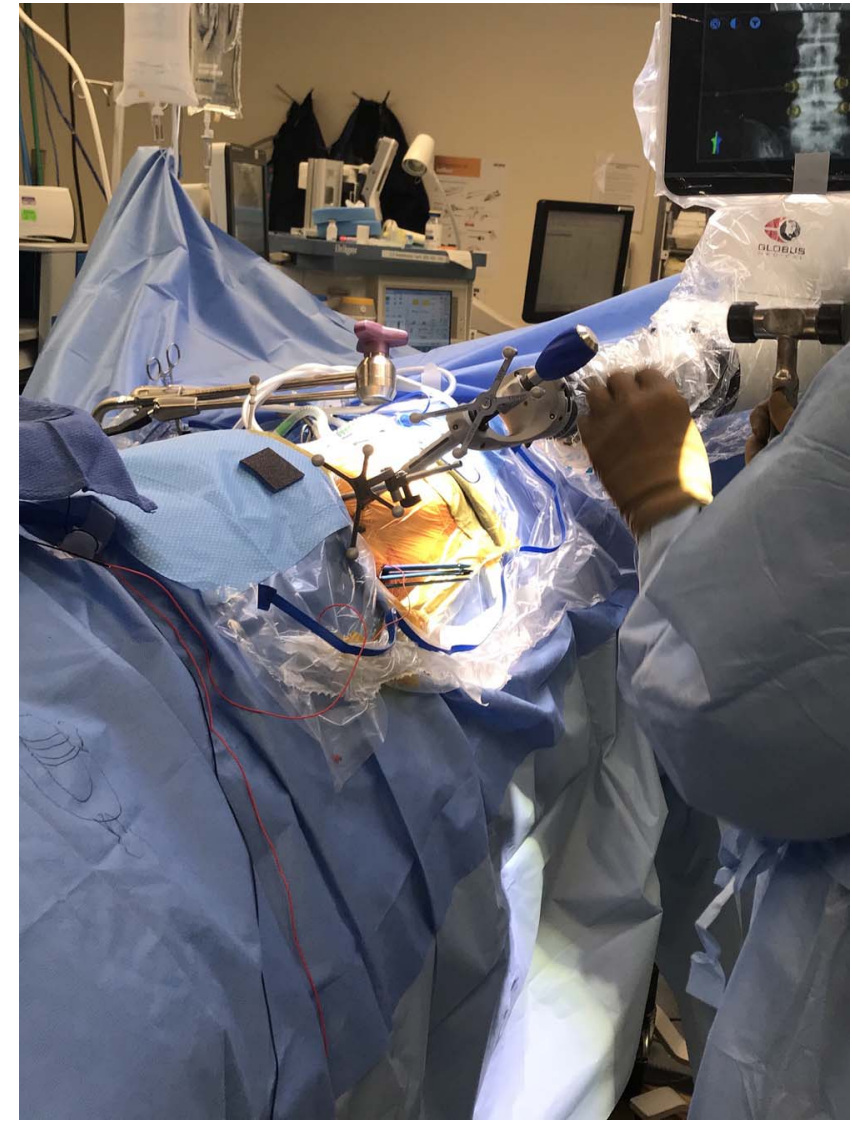

Figure 3. Intraoperative photo of robotic use for pedicle screw placement in the single position lateral. After completion of the LLIF portion, the table is tilted ventrally. The placement of the DRB, registration fluoroscopy, and screw drilling, tapping, and placement all proceed as in the prone position. The robot is brought in from the patient's dorsal side.

patients) were placed successfully using the robot $(99 \%)$. Those 5 pedicle screws were placed by converting to a percutaneous fluoro-guided technique without robotic assistance. Eighty-six patients had screws placed using a percutaneous technique, and 15 patients had screws placed using an open technique. A total of 98 patients underwent concurrent interbody placement: 28 ALIFs, 12 LLIFs, and 58 TLIFs. All ALIFs and LLIFs were performed prior to placement of the screws. Four of the 12 LIF patients had screws placed with the patient in the lateral position.

No patients had screw-related complications intraoperatively or postoperatively, and no patients returned to the operating room for screw revision.

Thirteen patients had postoperative CT scans for various reasons, some to check screw position in the setting of postoperative radiculopathy, and some unrelated to screw placement, for example postoperative ileus. This accounted for 66 screws. Sixtytwo screws were graded as an A, 4 screws were 
graded as a B; all 4 screws had a lateral breach $<2$ $\mathrm{mm}$. All $(100 \%)$ of screws were in an acceptable position.

\section{DISCUSSION}

In this case series of our initial experience with a novel navigated robotics system, we demonstrate that it can be used to place thoracolumbar screws safely in a variety of scenarios: open or percutaneous, after interbody placement, and in the prone or lateral position. It highlights the versatility of this device.

This system also has the potential to increase screw placement accuracy as demonstrated by zero screw-related complications, no return to the operating room for screw revision, and all screws in an acceptable position within the pedicle on subgroup analysis. Screw malposition can have devastating consequences, including vascular and neurologic complications, especially when normal anatomy may be distorted. ${ }^{6}$ Many prior studies have examined the accuracy of screw placement using computer-guided navigation versus freehand techniques. Accuracy rates are higher with computer-guided navigation, with computer guided navigation rates of $93.3 \%$ to $97.7 \%$, versus freehand rates of $86.6 \%$ to $91.3 \%$. $^{7-9}$

Robotic-guided systems have also shown promising results. Most prior studies have been performed using SpineAssist or Renaissance (Mazor Robotics Ltd, Caesarea, Israel), and demonstrate accuracy rates between $96.7 \%$ and $98.9 \% .^{10,11}$ The potential for perfecting accuracy is even higher when combining both navigation and robotics. Recently, there was 1 small case series published examining the use of ExcelsiusGPS in 2 patients, demonstrating $100 \%$ accuracy in screw placement in 8 screws. ${ }^{12}$ This finding is further confirmed with the present study demonstrating $100 \%$ accurate screw placement in the subgroup analysis of 66 screws. Similarly, a recent study examining the feasibility of another robotic-navigation guided system, Mazor X (Mazor Robotics Ltd) reported an accuracy rate of $98.7 \%$ examining 75 screws. ${ }^{13}$

In addition to improving accuracy, while not directly examined in this study, importantly the robotic-navigation system has the added advantage of likely reducing radiation exposure intraoperatively to the both the patient and the surgeon. The only fluoroscopy required intraoperatively are the few images required for registration, including a good AP and lateral at each instrumented level. This is especially valuable in cases with percutaneous screw placement, which generally require substantial fluoroscopy time. Prior studies have shown a significant decrease in radiation exposure with the use of robotics. ${ }^{14}$ It should be noted, however, that in the preoperative CT planning mode, the patient must undergo a CT scan and its associated radiation exposure. Nonetheless, many patients may undergo a preoperative CT scan for surgical planning purposes not related to the use of the robot.

The ExcelsiusGPS provides advantages over previously described robotic systems, namely obviating the need for K-wires. The Mazor SpineAssist and Renaissance systems rely on interspinous clamps for fiducial registration, and the robotic arms are then screwed onto the interspinous clamp. This may allow for inadvertent trajectory disruption with motion, and thus requires a K-wire initially to maintain position. The fiducial in the PSIS and the locked rigid arm in the ExcelsiusGPS system avoids this.

Although overall the use of the robotic-navigation technology was quite successful, it is important to note the 5 cases in which use of the robot was aborted all together, as described above. Furthermore, in the remaining cases in which the robot was used, 5 screws were converted to be placed manually. This was due to difficulty with registration at 1 vertebral body in 1 case and issues with skiving in another case. These examples highlight the need for adequate fluoroscopy and an accurate registration, which can be an issue in patients with severe obesity, osteopenia, or deformity. Difficulties with intraoperative fluoroscopy and registration have been similarly described with other robotic technology. ${ }^{10}$ In this study, 2D fluoroscopy was performed to register with a preoperative CT scan. It possible that use of intraoperative CT scan or $3 \mathrm{D}$ fluoroscopy may decrease problems with registration. Although the robot may enhance surgical technique, it is important that the surgeon retain the skills of more traditional methods in these scenarios. As the robot technology continues to evolve, we anticipate that the registration process will improve in these difficult cases.

Limitations of this study include lack of postoperative CT scan on all patients to more thoroughly assess accuracy radiographically, as well as lack of a control group. Future investigations will focus on 
assessing accuracy using direct comparison with manual screw placement.

In conclusion, in the largest description using the novel ExcelsiusGPS robot-navigation technology, we demonstrate its safety and efficacy in accurate placement of thoracolumbar pedicle screws, offering the potential to reduce screw-related complications.

\section{REFERENCES}

1. Rajaee SS, Bae HW, Kanim LE, Delamarter RB. Spinal fusion in the United States: analysis of trends from 1998 to 2008. Spine. 2012;37(1):67-76.

2. Gaines RW Jr. The use of pedicle-screw internal fixation for the operative treatment of spinal disorders. JBJS. 2000;82(10):1458-1476.

3. Amato V, Giannachi L, Irace C, Corona C. Accuracy of pedicle screw placement in the lumbosacral spine using conventional technique: computed tomography postoperative assessment in 102 consecutive patients. J Neurosurg Spine. 2010;12(3):306-313.

4. Joseph JR, Smith BW, Liu X, Park P. Current applications of robotics in spine surgery: a systematic review of the literature. Neurosurg Focus. 2017;42(5):E2.

5. Gertzbein SD, Robbins SE. Accuracy of pedicular screw placement in vivo. Spine. 1990;15(1):11-14.

6. Hicks JM, Singla A, Shen FH, Arlet V. Complications of pedicle screw fixation in scoliosis surgery: a systematic review. Spine. 2010;35(11):E465-E470.

7. Kosmopoulos V, Schizas C. Pedicle screw placement accuracy: a meta-analysis. Spine. 2007;32(3):E111-E120.

8. Verma R, Krishan S, Haendlmayer K, Mohsen A. Functional outcome of computer-assisted spinal pedicle screw placement: a systematic review and meta-analysis of 23 studies including 5, 992 pedicle screws. Eur Spine J. 2010;19(3):370375.

9. Aoude AA, Fortin M, Figueiredo R, Jarzem P, Ouellet J, Weber MH. Methods to determine pedicle screw placement accuracy in spine surgery: a systematic review. Eur Spine J. 2015;24(5):990-1004

10. Hu X, Ohnmeiss DD, Lieberman IH. Robotic-assisted pedicle screw placement: lessons learned from the first 102 patients. Eur Spine J. 2013;22(3):661-666.

11. Keric N, Doenitz C, Haj A, et al. Evaluation of robotguided minimally invasive implantation of 2067 pedicle screws. Neurosurg Focus. 2017;42(5):E11.

12. Jiang B, Ahmed AK, Zygourakis CC, et al. Pedicle screw accuracy assessment in ExcelsiusGPS ${ }^{\circledR}$ robotic spine surgery: evaluation of deviation from pre-planned trajectory. Chinese Neurosurg J. 2018;4(1):23.

13. Khan A, Meyers JE, Siasios I, Pollina J. Next-generation robotic spine surgery: first report on feasibility, safety, and learning curve. Oper Neurosurg (Hagerstown). 2019;17(1):6169.

14. Lieberman I, Hardenbrook M, Wang J, Guyer RD, Khanna AJ. P152. Radiation exposure using miniature robotic guidance for spinal surgery. Spine J. 2007;7(5)(suppl):152S$153 \mathrm{~S}$.

Disclosures and COI: Two authors receive consulting money from a company involved in the manufacture of a technology examined in this study.

Corresponding Author: Deeptee Jain, MD, 301 E 17th St, Suite 1402, New York, NY 10003. Phone: (414) 364-7762; Email: deeptee.jain@gmail. com.

Published 31 October 2019

This manuscript is generously published free of charge by ISASS, the International Society for the Advancement of Spine Surgery. Copyright (c) 2019 ISASS. To see more or order reprints or permissions, see http://ijssurgery.com. 